\title{
Optimization of a Microgrid Integrated with Renewable Energies using PSO
}

\author{
Navid Salehi ${ }^{1}$, Herminio Martínez-García ${ }^{1, *}$, Guillermo Velasco-Quesada ${ }^{1}$, and Encarna \\ García-Vílchez ${ }^{1}$ \\ ${ }^{1}$ Electronic Engineering Department, Eastern Barcelona School of Engineering (EEBE), Technical \\ University of Catalonia (UPC) - BarcelonaTech, Barcelona, Spain
}

\begin{abstract}
Nowadays, microgrids (MGs) play a crucial role in modern power systems due to possibility of integrating renewable energies into the MG system. It is expected in near future collaborating microgrids as a community has a great affect in providing efficient energy for suppliers. Therefore, optimal operation of a MG, as a first step, is an important issue in this field. In this paper, by considering an integrated MG comprised of conventional generators, photovoltaic and wind a particle swarm optimization (PSO) is applied to minimize the cost function of the MG. Finally, the simulation results are discussed and analyzed to evaluate the system operation.
\end{abstract}

\section{Introduction}

Microgrids (MGs) can be consider as a small power system which are combined with distributed generations (DGs), energy storage systems (ESSs), and loads [1]. Moreover, DGs can produce energy by different types of generators such as diesel generators or renewable source of energy such as solar or wind energy. Therefore, MGs can be integrated with renewable energies (REs) to take advantage of limitless sources of energy with no environmental pollution as a vital commitment to the future of the world [2].

As it can be inferred, an MG can produce and consume energy according to the instantaneous generated energy and load demands. Therefore, the power balance in MGs is more critical due to higher dynamic of the system in comparison with power system. This issue will be more acute when the MG is integrated with REs due to uncertainty, inherent randomness and volatility of renewable generators [3]. As a consequence, energy storage system (EES) plays a vital role in order to provide the power balance especially in standalone operation mode.

Batteries, as one of the most common parts of ESSs, can effectively cope with the energy swing in a single MG owing to their high dynamic response to absorb or exude of energy [4]. However, in grid-connected MGs, power system can play the same role of batteries in ESS with more reliability and economically [5] then the importance of ESS reduce significantly. Grid-connected MGs can exchange energy with power system, therefore, the paradigm of conventional power system will be affected by injecting energy to the power system, and it makes some changes in the adjustment related to the power system [6].

Microgrid community is another effective method to reduce the impact of ESS in the single MG. In MG community, energy can exchange between each other in order to provide the power balance [7]. In this case, the setting of the power system will be affected less than a

\footnotetext{
* Corresponding author: herminio.martinez@upc.edu
} 
single MG.Energy management system (EMS) in MG community plays a significant role in order to realize some features such as: managing the power flow between MGs and power system [8], optimal operation [9], generation prediction for all RES [10]. One on the most important tasks of an EMS, is realizing the optimal operation of each individual MG in order to determine how much energy can exchange optimally by considering the practical constraints of a particular MG.

There are various optimization methods such as evolutionary programing, dynamic programing, genetic algorithm, and artificial neural network approaches, in order to optimize the operation of the system by considering the equality and inequality constraints [11], [12]. In this paper, particle swarm optimization (PSO) is applied to a single MG integrated with RES in order to minimize the operational cost of the system. In PSO each individual particle makes his decision using his own experience together with other individuals' experiences [13]. The individual particles are drawn stochastically toward the position of present velocity of each individual, their own previous best performance, and the best previous performance of their neighbors [14]. The main advantages of the PSO algorithm can be elaborated as: simple concept, easy implementation, robustness to control parameters, and computational efficiency.

In this paper, after determining the configuration of the proposed MG, a particular case or scenario, the cost function, equality and inequality constraints of the power generations will be defined, and the system will be optimized by applying a PSO algorithm. The optimizer determines the economic power generation of all generators based on the load profile. Moreover, the probable surplus energy or required energy from power system can be calculated over this optimization. Finally, the simulation results will be presented in order to evaluate the system operation.

\section{System Configuration}

As it can be seen from Fig. 1, the considered single MG in order to study in this paper, as a particular scenario, comprises a photovoltaic (PV) power plant, a wind turbine (WT) generator, three conventional diesel generators (G1-G3), and loads which all are connected to the power system (PS). On the one hand, the power profile of the load, over a 24-hour interval, is shown in Fig. 2. On the other hand, Fig. 3 and Fig. 4 show the generated power of photovoltaic, and wind turbine, respectively, also over a 24 -hour interval. In addition, the nominal power for each element included in Fig. 1 is listed in Table I.

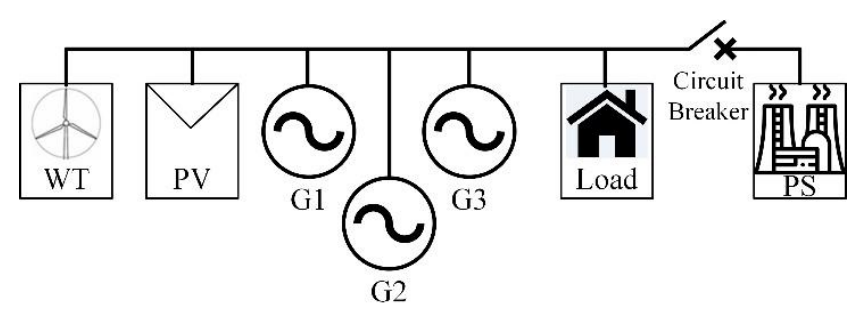

Fig. 1. Block diagram of the considered single MG. It comprises a photovoltaic (PV) power plant, a wind turbine (WT) generator, three conventional diesel generators (G1-G3), and loads which all are connected to the power system (PS).

The configuration in Fig.1 with three diesel generators and without any energy storage system which all are connected to the main grid is usual in some countries which their weak main grid system cannot guarantee to supply the load at all moments or maybe for some hours a day the main grid is cut-off. Therefore, as can be seen from Fig. 1, the circuit breaker can 
connect the MG to the main grid in case of either existing surplus energy or shortage energy of energy in MG.

In the following, the characteristics of each power generation and load demand is elaborated, and the cost function for each part is described. Then, the optimization problem is defined based on the cost function and operation function of each power generation.

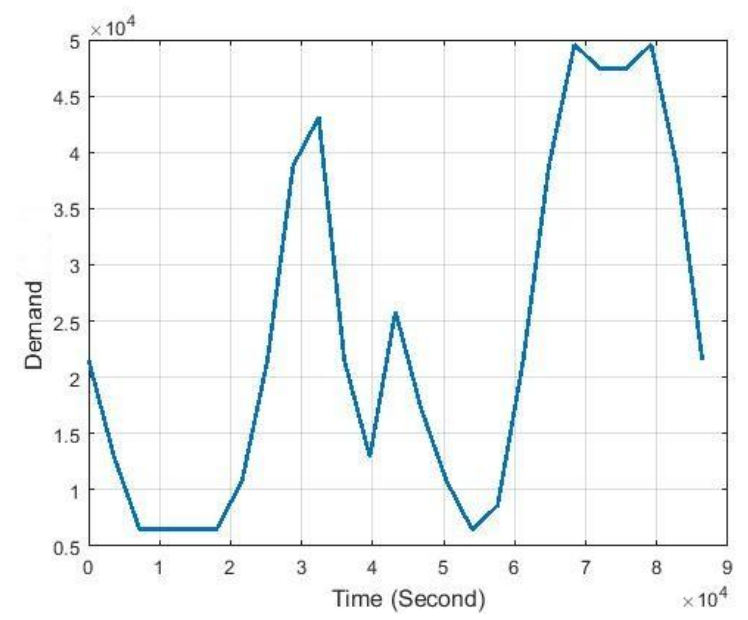

Fig. 2. Load's power profile.

Table 1. Nominal power of the units

\begin{tabular}{|c|c|c|c|}
\hline $\begin{array}{c}\text { Power generation } \\
\text { and load demand }\end{array}$ & $\begin{array}{c}\text { Nominal } \\
\text { power }\end{array}$ & $\begin{array}{c}\text { Minimum } \\
\text { power }\end{array}$ & $\begin{array}{c}\text { Maximum } \\
\text { power }\end{array}$ \\
\hline Photovoltaic & $5 \mathrm{~kW}$ & 0 & $5 \mathrm{~kW}$ \\
\hline Wind & $5 \mathrm{~kW}$ & 0 & $5 \mathrm{~kW}$ \\
\hline Diesel generator 1 & $10 \mathrm{~kW}$ & $2 \mathrm{~kW}$ & $10 \mathrm{~kW}$ \\
\hline Diesel generator 2 & $25 \mathrm{~kW}$ & $4 \mathrm{~kW}$ & $25 \mathrm{~kW}$ \\
\hline Diesel generator 3 & $15 \mathrm{~kW}$ & $3 \mathrm{~kW}$ & $15 \mathrm{~kW}$ \\
\hline Load & ------ & $0.6 \mathrm{~kW}$ & $50 \mathrm{~kW}$ \\
\hline
\end{tabular}

\subsection{Photovoltaic Generation}

Fig. 3 shows the solar irradiation profile, and for this study it is supposed that the PV panels are implemented on $100 \mathrm{~m}^{2}$ of area. Due to the high cost of production especially for solar and wind energies, usually a maximum power point tracking (MPPT) system is implemented for these systems to be sure that the maximum possible power is extracting from the implemented system. However, the cost function of solar generation is defined in (1) by considering the investment cost of the equipment and operation and maintenance (O\&M) cost of the energy generation [15].

where,

$$
C F\left(P_{P V}\right)=\alpha I_{P V}^{P} P_{P V}+G_{P V}^{E} P_{P V}
$$




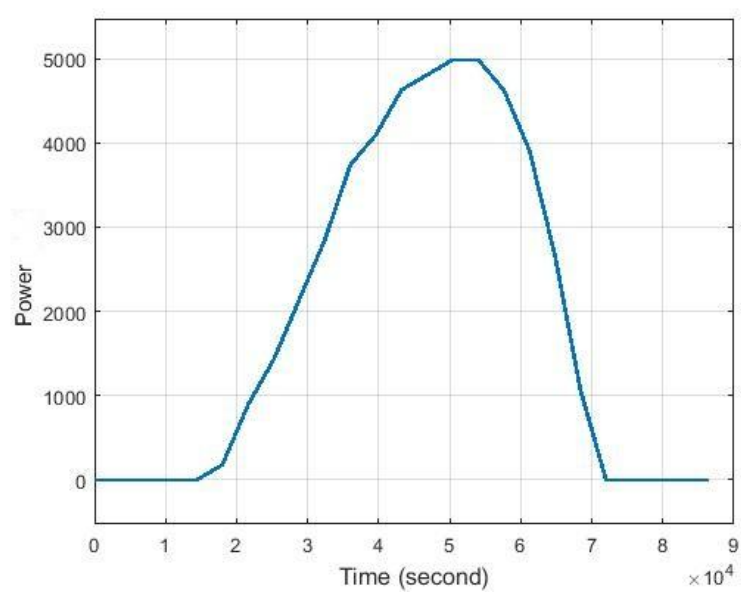

Fig. 3. PV power profile.

$$
\alpha=\frac{r}{\left[1-(1+r)^{-N}\right]}
$$

where $P_{P V}$ is solar power $(k W), \alpha$ is annuitization coefficient, $r$ is interest rate, $N$ is investment lifetime, $I^{P}$ is investment costs, and $G^{E}$ is O\&M costs for solar generation.

As it can be inferred from the solar radiation, it is obvious the power generation of photovoltaic panels can be between 0 and maximum value:

$$
0 \leq P_{P V} \leq P_{P V}^{\max }
$$

\subsection{Wind Generation}

Fig. 4 shows the wind profile for this study. The wind turbine trips from the system when the wind speed exceeds the maximum wind value, until the wind gets back to its nominal value. The same as solar energy, wind energy also takes the advantage of MPPT in order to harvest the maximum possible power for each time. Moreover, the cost function of wind generation only by considering the investment cost of the equipment and O\&M cost of the energy generation is defined below [15]:

$$
C F\left(P_{W T}\right)=\alpha I_{W T}^{P} P_{W T}+G_{W T}^{E} P_{W T}
$$

where $\alpha$ is defined in (2), $I^{P}$ is investment costs and $G^{E}$ is O\&M costs for wind generation. The wind turbine generation has the following restriction:

$$
0 \leq P_{W T} \leq P_{W T}^{\max }
$$

\subsection{Diesel Generators}

The conventional generators are more reliable and predictable in an MG rather than solar and wind generation. The cost function of DGs usually is considered as a second order polynomial function as in (6). 


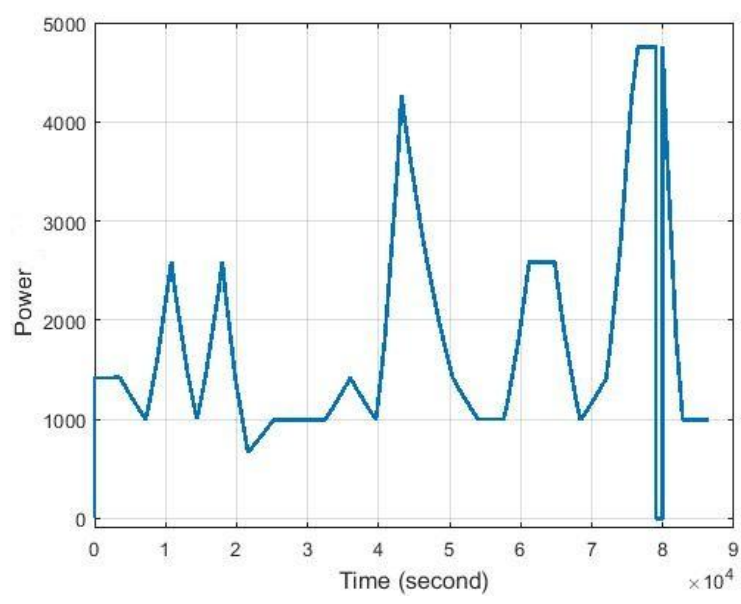

Fig. 4. Wind power profile.

$$
C F\left(P_{D G}\right)=\alpha_{D G}+\beta P_{D G}+\gamma P_{D G}^{2}
$$

Diesel generators have lower and upper limit of power generation, and they have to produce energy in the permitted range. However, an optimizer can set the DGs generation to the optimum value with lowest cost meanwhile the restrictions of the system are satisfied:

$$
P_{D G}^{\min } \leq P_{D G} \leq P_{D G}^{\max }
$$

\subsection{Power System}

The proposed MG in this paper is connected to the main grid. Therefore, if the power generation units in the MG are not able to supply the load, main grid compensate the energy. Moreover, if the produced power in the MG is more than load power, the surplus energy can transfer to the main grid. In order to calculate the cost of energy transaction, $C F\left(P_{P S}\right)$ the following equation can be used:

$$
C F\left(P_{P S}\right)= \pm P_{P S} \Delta t
$$

where $\Delta t$ is the period that the power $P_{P S}$ is injected to the grid (with negative sign) or is absorbed from the grid (with positive sign). In addition, there is no any power limit for the main grid.

\section{Optimization Problem}

The optimization problem in this paper is a real time operation optimization of the MG in order to minimize the defined cost function by considering the constraints of the power generation units. The objective function $(O F)$ for this problem is the sum of all cost functions subject to the aforementioned constraints of the units.

$$
O F=\sum\left(C F_{D G 1}+C F_{D G 2}+C F_{D G 3}+C F_{P S}\right)
$$


s.t:

- PV generation constraints in (3);

- WT generation constraints in (5);

- DGs constraints in (7);

and:

$$
P_{D G 1}+P_{D G 2}+P_{D G 3}=P_{L o a d}-P_{P V}-P_{W T}\left(+/-P_{P S}\right)
$$

where $+/-P_{P S}$ represents the surplus energy transferred (positive) to the main grid or consumed (negative) from it.

Fig. 5 shows the optimization procedure for this study. As it can be observed, the MG is simulated by MATLAB-Simulink ${ }^{\circledR}$, and, in order to optimize the MG operation, the data of MG transfers to the optimizer. In this paper, in order to optimize the problem a PSO algorithm is applied to the problem. PSO determines the economic power generation for each diesel generator at the end of the each calculation. For this purpose, PSO minimize the objective function by considering the constraints which are defined in system model. The first priority is supply the load by the power generation units of the MG. However, if the load requires more energy, power system can supply it. On the other hand, if the power generation of the MG is more than the load consume, the surplus energy is transferred to the main grid.

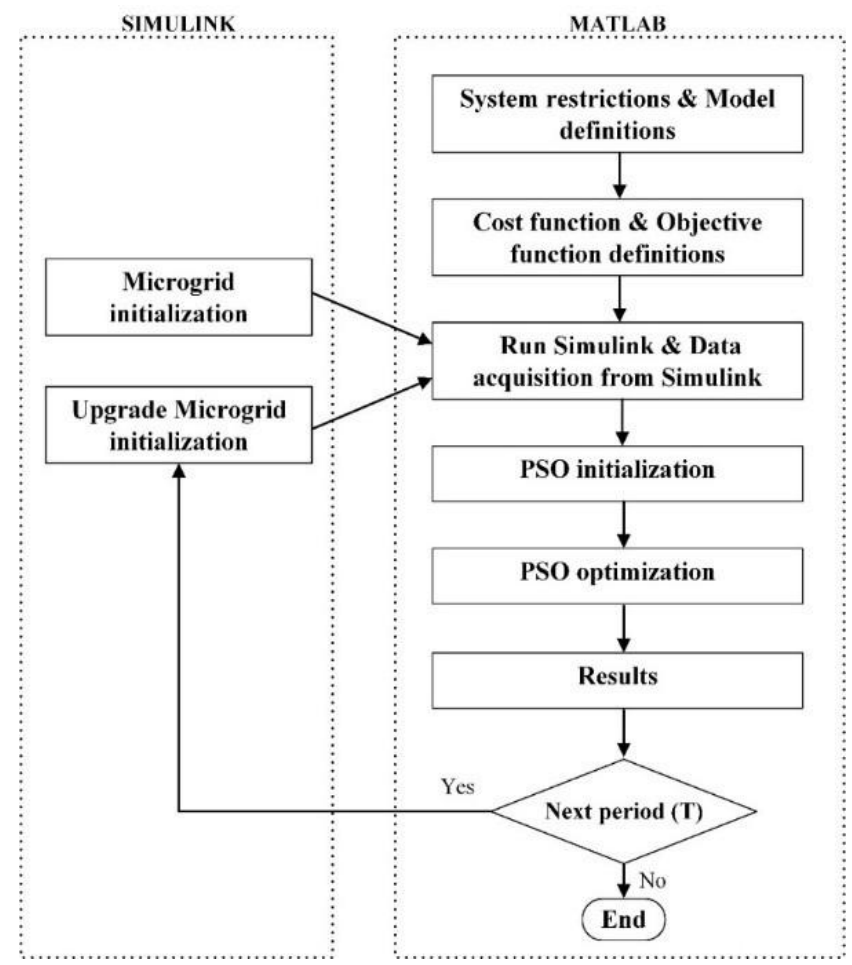

Fig. 5. Optimization procedure by PSO. 


\section{Simulation Results}

A simulation carried out on an MG with the configuration of Fig. 1 and power rate that is shown in Table 1. The PSO take the real time power of the PV, wind and load of the MG and based on these information optimize the power generation of the DGs in order to operate at lowest cost. The shortage and surplus energies of the MG can be traded off with the main grid through the power system. Moreover, the power losses of transmission lines are considered in this simulation to have more accurate results. In (11) the power loss equation is presented. In order to calculate the transmission line losses based on the following equation, the $B$ loss coefficient are listed in (12)-(14).

$$
P_{L}=\sum_{i=1}^{3} \sum_{j=1}^{3} P_{i}+B_{i j} P_{j}+\sum_{i=1}^{3} B_{0 i} P_{i}+B_{00}
$$

where,

$$
\begin{aligned}
\mathrm{B}_{i j} & =\left[\begin{array}{lll}
0.0017 & 0.0012 & 0.0007 \\
0.0012 & 0.0014 & 0.0009 \\
0.0007 & 0.0009 & 0.0031
\end{array}\right] \\
\mathrm{B}_{0 i} & =\left[\begin{array}{lll}
-0.3908 & -0.1279 & 0.7047
\end{array}\right] \\
\mathrm{B}_{00} & =0.056
\end{aligned}
$$

Moreover, in order to calculate the cost of each generation units the parameters in Tables 2 and 3 are used in this simulation according to the corresponding equation that defined in section 2 for the PV, wind and DGs units.

Table 2. PV and WT parameters

\begin{tabular}{|c|c|}
\hline \multicolumn{2}{|c|}{ Power generation unit } \\
\hline PV Generator & Wind Turbine \\
\hline$I_{P V}=5000(\$ / \mathrm{kW})$ & $I_{W T}=1400(\$ / \mathrm{kW})$ \\
\hline$G_{P V}=1.6(\$ / \mathrm{kW})$ & $G_{W T}=1.6(\$ / \mathrm{kW})$ \\
\hline$r_{P V}=0.09$ & $r_{W T}=0.09$ \\
\hline$N_{P V}=20$ & $N_{W T}=20$ \\
\hline
\end{tabular}

Table 3. DGs coefficients.

\begin{tabular}{|c|c|c|c|}
\hline DG unit & $\boldsymbol{\alpha}$ & $\boldsymbol{\beta}$ & $\boldsymbol{\gamma}$ \\
\hline 1 & 220 & 8.5 & 0.009 \\
\hline 2 & 225 & 8 & 0.008 \\
\hline 3 & 210 & 9 & 0.009 \\
\hline
\end{tabular}

As it can be seen in Fig 5, the model of each power generation unit are defined in SIMULINK, and the cost function, objective function and constraints of power generation units are defined in MATLAB environment, therefore, MATLAB and SIMULINK effectively interchange their data. Moreover, the PSO parameters are listed in Table 4. 
Table 4. PSO parameters

\begin{tabular}{|c|c|}
\hline Parameter & Value \\
\hline Max number of iteration & 50 \\
\hline Population size (swarm size) & 200 \\
\hline Inertia weight & 1 \\
\hline Inertia weight damping ratio & 0.99 \\
\hline Personal learning coefficient & 2 \\
\hline Global learning coefficient & 2 \\
\hline
\end{tabular}

Finally, Table 5 shows the optimization results of the MG. In order to avoid generating enormous data, the step time of optimization is adjusted for every 90 minutes for a whole day.

Table 5. Simulation results.

\begin{tabular}{|c|c|c|c|c|c|c|}
\hline $\begin{array}{c}{\left[\begin{array}{c}\text { Pdg1 Pdg2 Pdg3 }] \\
(\mathbf{k W})\end{array}\right.}\end{array}$ & $\begin{array}{c}\text { Total } \\
\text { generated } \\
\text { power } \\
(\mathbf{k W}) \\
\end{array}$ & $\begin{array}{l}\text { Total cost } \\
\text { (per unit) }\end{array}$ & $\begin{array}{c}\text { Power } \\
\operatorname{loss}(W)\end{array}$ & $\begin{array}{c}\text { Load } \\
\text { Power } \\
(k W)\end{array}$ & $\begin{array}{c}\text { Grid } \\
\text { Power } \\
(k W)\end{array}$ & $\begin{array}{l}\text { Time } \\
(\text { min) }\end{array}$ \\
\hline$\left[\begin{array}{lllll}6.922 & 7.813 & 6.884\end{array}\right]$ & 21.620 & 1530.191 & 61 & 21.558 & 0 & 0 \\
\hline$\left[\begin{array}{llll}6.853 & 7.739 & 6.810\end{array}\right]$ & 21.574 & 1501.526 & 60 & 21.513 & 0 & 90 \\
\hline$\left[\begin{array}{lll}2 & 4 & 3\end{array}\right]$ & 9.182 & 321.655 & 11 & 6.467 & -2.703 & 180 \\
\hline$\left[\begin{array}{lll}2 & 4 & 3\end{array}\right]$ & 9.418 & 321.655 & 11 & 6.467 & -2.939 & 270 \\
\hline $\left.\begin{array}{llll} & 9.290 & 10.518 & 9.241\end{array}\right]$ & 30.235 & 2677.520 & 111 & 30.124 & 0 & 360 \\
\hline 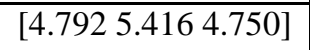 & 17.448 & 771.946 & 29 & 17.419 & 0 & 450 \\
\hline 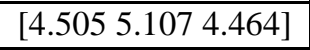 & 17.272 & 690.824 & 26 & 17.246 & 0 & 540 \\
\hline$\left[\begin{array}{lll}2 & 4 & 3\end{array}\right]$ & 12.051 & 321.655 & 11 & 7.070 & -4.969 & 630 \\
\hline$\left[\begin{array}{llll}9.999 & 14.394 & 12.680\end{array}\right]$ & 38.985 & 4319.668 & 184 & 38.800 & 0 & 720 \\
\hline$\left[\begin{array}{llll}10 & 22.206 & 15\end{array}\right]$ & 47.717 & 7268.401 & 293 & 47.423 & 0 & 810 \\
\hline$\left[\begin{array}{lll}10,24.216 & 15\end{array}\right]$ & 49.787 & 8030.820 & 316 & 49.470 & 0 & 900 \\
\hline$\left[\begin{array}{llll}10 & 24 & 16\end{array}\right]$ & 50 & 8102.655 & 317 & 49.472 & 0 & 990 \\
\hline$\left[\begin{array}{llll}10 & 26 & 17\end{array}\right]$ & 53 & 9216.655 & 352 & 49.578 & -3.07 & 1080 \\
\hline$\left[\begin{array}{llll}9.999 & 22.567 & 15\end{array}\right]$ & 47.567 & 7400.486 & 297 & 47.269 & 0 & 1170 \\
\hline$\left[\begin{array}{llll}10 & 21.997 & 15\end{array}\right]$ & 47.559 & 7192.882 & 291 & 47.268 & 0 & 1260 \\
\hline
\end{tabular}

As it can be seen from Table 5 at the times 180, 270, 630 and 1080 min., the generated power by MG is more than load profile at these times. Therefore, the surplus energies are transferred to the main grid by means of the power system. The power losses in this simulation just considered the loss power between diesel generators and as it can be observed by increasing the generated power by each DG the power loss increase as well. Total costs include the cost of each DG, PV and wind turbine based on the cost functions in (6), (1) and (4), respectively. The costs of the PV and wind turbine are calculated based on the maximum power which they can produce at the time. The costs of the diesel generators are calculated based on the optimum power which is calculated by the PSO optimizer. In order to evaluate the cost function of the power generation units in the MG, the parameter's values are defined in Tables 2 and 3. 


\section{Conclusions}

In this paper, an optimization procedure for an integrated grid-connected MG comprised of renewable energies has studied. The PSO optimization applied to the proposed MG to optimize the power generation of diesel generators by minimizing their cost functions and considering the constraints related to DGs, PV and wind energies. In order to satisfy the power balance in the MG, the main grid can absorb or inject energy to the MG. The optimizer applies to the MG for the defined time interval to keep the MG operation optimum and economic.

Finally, the simulation results show the optimum power values for diesel generators; however, the generated power of PV and WT are the maximum power which they can produce at the time based on solar irradiation and speed of the wind. The generated power by PV and WT affect the power balance equation and consequently to the optimum values of DGs generation.

\section{Acknowledgment}

This work has been partially supported by the Spanish Ministerio de Ciencia, Innovación y Universidades (MICINN)-Agencia Estatal de Investigación (AEI) and the European Regional Development Funds (FEDER), by project PGC2018-098946-B-I00.

\section{References}

1. Montuori, Lina, Manuel Alcázar-Ortega, Carlos Álvarez-Bel, and Alex Domijan. "Integration of renewable energy in microgrids coordinated with demand response resources: Economic evaluation of a biomass gasification plant by Homer Simulator." Applied Energy 132 (2014): 15-22.

2. Manjili, Yashar Sahraei, Amir Rajaee, Mo Jamshidi, and Brian T. Kelley. "Intelligent decision making for energy management in microgrids with air pollution reduction policy." In 2012 7th International Conference on System of Systems Engineering (SoSE), pp. 13-18. IEEE, 2012.

3. Wang, Ran, Ping Wang, Gaoxi Xiao, and Shimin Gong. "Power demand and supply management in microgrids with uncertainties of renewable energies." International Journal of Electrical Power \& Energy Systems 63 (2014): 260-269.

4. Atia, Raji, and Noboru Yamada. "Sizing and analysis of renewable energy and battery systems in residential microgrids." IEEE Transactions on Smart Grid 7, no. 3 (2016): 1204-1213.

5. Malysz, Pawel, Shahin Sirouspour, and Ali Emadi. "An optimal energy storage control strategy for grid-connected microgrids." IEEE Transactions on Smart Grid 5, no. 4 (2014): 1785-1796.

6. Schwaegerl, Christine, Liang Tao, Pierluigi Mancarella, and Goran Strbac. "Can microgrids provide a new paradigm for network operation? an evaluation of their technical, commercial and environmental benefits." In 20th International CIRED Conference and Exhibition. 2009.

7. Tian, Peigen, Xi Xiao, Kui Wang, and Ruoxing Ding. "A hierarchical energy management system based on hierarchical optimization for microgrid community economic operation." IEEE Transactions on Smart Grid 7, no. 5 (2015): 2230-2241.

8. Al-Saedi, Waleed, Stefan W. Lachowicz, Daryoush Habibi, and Octavian Bass. "Power flow control in grid-connected microgrid operation using Particle Swarm Optimization under variable load conditions." International Journal of Electrical Power \& Energy Systems 49 (2013): 76-85. 
9. Khodaei, Amin, and Mohammad Shahidehpour. "Optimal operation of a communitybased microgrid." In 2011 IEEE PES Innovative Smart Grid Technologies, pp. 1-3. IEEE, 2011.

10. Pereira, Mario, D. Munoz De La Pena, and Daniel Limón. "Robust economic model predictive control of a community micro-grid." Renewable Energy 100 (2017): 3-17.

11. Park, Young-Moon, Jong-Ryul Won, and Jong-Bae Park. "A new approach to economic load dispatch based on improved evolutionary programming." Engineering Intelligent Systems for Electrical Engineering and Communications 6, no. 2 (1998): 103-110.

12. Walters, David C., and Gerald B. Sheble. "Genetic algorithm solution of economic dispatch with valve point loading." IEEE transactions on Power Systems 8, no. 3 (1993): 1325-1332.

13. Yoshida, Hirotaka, Kenichi Kawata, Yoshikazu Fukuyama, Shinichi Takayama, and Yosuke Nakanishi. "A particle swarm optimization for reactive power and voltage control considering voltage security assessment." IEEE Transactions on power systems 15, no. 4 (2000): 1232-1239.

14. Clerc, Maurice, and James Kennedy. "The particle swarm-explosion, stability, and convergence in a multidimensional complex space." IEEE transactions on Evolutionary Computation 6, no. 1 (2002): 58-73.

15. Fueyo, Norberto, Yosune Sanz, Marcos Rodrigues, Carlos Montañés, and César Dopazo. "The use of cost-generation curves for the analysis of wind electricity costs in Spain." Applied energy 88, no. 3 (2011): 733-740. 